\title{
Multiscale Fragile Watermarking Based on the Gaussian Mixture Model
}

\author{
Hua Yuan and Xiao-Ping Zhang, Senior Member, IEEE
}

\begin{abstract}
In this paper, a new multiscale fragile watermarking scheme based on the Gaussian mixture model (GMM) is presented. First, a GMM is developed to describe the statistical characteristics of images in the wavelet domain and an expectation-maximization algorithm is employed to identify GMM model parameters. With wavelet multiscale subspaces being divided into watermarking blocks, the GMM model parameters of different watermarking blocks are adjusted to form certain relationships, which are employed for the presented new fragile watermarking scheme for authentication. An optimal watermark embedding method is developed to achieve minimum watermarking distortion. A secret embedding key is designed to securely embed the fragile watermarks so that the new method is robust to counterfeiting, even when the malicious attackers are fully aware of the watermark embedding algorithm. It is shown that the presented new method can securely embed a message bit stream, such as personal signatures or copyright logos, into a host image as fragile watermarks. Compared with conventional fragile watermark techniques, this new statistical model based method modifies only a small amount of image data such that the distortion on the host image is imperceptible. Meanwhile, with the embedded message bits spreading over the entire image area through the statistical model, the new method can detect and localize image tampering. Besides, the new multiscale implementation of fragile watermarks based on the presented method can help distinguish some normal image operations such as JPEG compression from malicious image attacks and, thus, can be used for semi-fragile watermarking.
\end{abstract}

Index Terms-Authentication, watermarking.

\section{INTRODUCTION}

W ITH the advancement of the multimedia storage, transmission technologies and the development of the World Wide Web, people are able to handle an increasing amount of digital information over the Internet. However, "seeing is no longer believing" [1]. Digital multimedia content is easily reproduced and modified without any trace of manipulations. Therefore, authentication techniques are required in applications where verification of integrity and authenticity of an image is essential [1], [2].

Digital watermarking provides a possible solution to the above problem, since it makes possible to identify the author of an image by embedding secret information in it. Authentication watermarks, also known as fragile watermarks, can be used to

Manuscript received March 26, 2005; revised January 2, 2006. This work was supported in part by the Canadian NSERC under Grant RGPIN239031 and in part by MICRONET. The associate editor coordinating the review of this manuscript and approving it for publication was Dr. Benoit Macq.

The authors are with the Department of Electrical and Computer Engineering, Ryerson University, Toronto, ON M5B 2K3 Canada (e-mail: hyuan@ee.ryerson.ca; xzhang@ee.ryerson.ca).

Digital Object Identifier 10.1109/TIP.2006.877310 detect any unauthorized alterations in an image. The fragile watermarks can be embedded in either the space domain or the transformed domain of an image. With the focus on the space domain, several fragile watermarking methods that utilize the least significant bit (LSB) of image data were developed. For example, a technique that inserts a checksum determined by the seven most significant bits into the LSBs of selected pixels was proposed in [3]. A technique which embeds a digital signature of the most significant bits of an image block into the least significant bits of the same block on a secret user key basis was developed in [4]. The technique in [4] is further extended to a public key scheme [5]. With the focus on the transformed domain, a wavelet-based fragile watermarking method that uses an optimal quantization step to detect image tampering is presented in [6]. The nature of multiresolution discrete wavelet decomposition allows the method to have spatial and frequency localization of image tampering. Noting the constraints of the single fragile watermark, researchers developed a hybrid authentication watermarking consisting of a fragile and a robust watermark [7]. For semi-fragile watermarking applications, a technique [8] that accepts some acceptable operations such as JPEG compression and reasonable brightness adjustment on watermarked images and rejects manipulations due to malicious attacks is developed using the authentication signature generated from the invariant properties of DCT coefficients [9]. Note that, in [9], the relationship of randomly selected blocks in the DCT domain is used to form the image signature. A similar idea was also employed in [10], where the relationship of randomly selected blocks is formed in the DCT domain to carry a robust watermark message. Meanwhile, a generic content-based approach is proposed in the wavelet domain to extract invariant features from image contents and then sign and embed them back into the image as watermarks for semi-fragile purposes [11].

While successful in detecting and localizing image tampering, the above-mentioned methods generally need to modify a large amount of image data to embed personalized watermark messages, and the modification may reduce the quality of the watermarked image. Also, the security of the watermark is of great concern, especially when a malicious attacker knows the watermarking algorithm. Therefore, an efficient secure fragile watermarking scheme that is capable of tampering detection and localization without modifying much image data is still highly desired.

In this paper, a novel multiscale fragile watermarking method that embeds watermarks at multiscale wavelet subspaces is presented, based on statistical modeling of the image in the wavelet domain. First, a two component Gaussian mixture 
model (GMM) is developed to describe the statistical characteristics of images in the wavelet domain. The GMM model is a compact representation of the wavelet coefficients of an image. In the new fragile watermarking embedding scheme, the wavelet multiscale subspaces are divided into watermarking blocks and the GMM model for each block is identified by an expectation-maximization (EM) algorithm. Then, the GMM model parameters of different watermarking blocks are adjusted to form certain relationships, which are employed to code fragile watermark messages for authentication, by modifying a few wavelet coefficients. The optimal wavelet coefficient modification scheme is developed to minimize the watermarking distortion. To securely embed the fragile watermarks, a secret embedding key is designed so that the new method is robust to watermark counterfeiting, even when the attackers have full knowledge of the watermark embedding algorithm. The secret embedding key can be encrypted and embedded as a robust watermark into the same host image of the fragile watermarks so that the decoding of fragile watermarks only requires a single encryption key other than the image itself.

The presented statistical model based new method can embed personal authentication signatures or copyright logos into the host image through modifying only few image data, while being able to detect and localize even very slight image tampering. The statistical model parameters are related to all data in a protected area and the multiscale modification of the data allows us to adjust the model parameters for watermarking with minimum host data modification. The new method is generally invulnerable to watermark counterfeiting due to the secret algorithm parameters and the secret embedding key that can be randomly selected and are kept unknown to potential attackers. The new method also provides a novel semi-fragile watermarking scheme that can be used to distinguish some normal image operations such as JPEG compression from malicious attacks. Numerical examples are given to demonstrate the effectiveness of the presented method.

The rest of the paper is organized as follows. In Section II, a GMM and its related EM algorithm are presented for image modeling in the wavelet domain. In Section III, the new fragile watermarking method based on the GMM and a secret embedding key scheme is developed. Experiments are conducted in Section IV to demonstrate the effectiveness of the proposed method. Section V concludes the paper.

\section{IMAGE MODELING USING THE GMM AND AN EM ALGORITHM}

The presented fragile watermarking method is based on image modeling using the GMM in the wavelet domain. The GMM parameters are then used for watermark embedding by the modification of selected wavelet coefficients.

The two-dimensional (2-D) discrete wavelet transform (DWT) is known to decompose an image into many wavelet subspaces at different scales. In each decomposed wavelet subspace, the wavelet coefficients have a peaky, heavy-tailed marginal distribution [12] and a near zero mean. Let $w_{i}$, $i=1, \ldots, K$, represent the wavelet coefficients in a single wavelet subspace. Its probability density function (PDF) $p\left(w_{i}\right)$ can be well modeled through a multistate Gaussian mixture

$$
p\left(w_{i}\right)=\sum_{m=1}^{M} P_{m} \cdot g\left(w_{i}, 0, \sigma_{m}^{2}\right)
$$

where the states of coefficients are represented by subscript " $m$," and the a priori probabilities of the $M$ states are represented by $P_{m}$. Note that

$$
\sum_{m=1}^{M} P_{m}=1
$$

and function $g\left(w_{i}, 0, \sigma_{m}^{2}\right)$ is a Gaussian PDF with distribution $N\left(0, \sigma_{m}^{2}\right)$

$$
g\left(w_{i}, 0, \sigma_{m}^{2}\right)=\frac{1}{\sqrt{2 \pi} \sigma_{m}} e^{-w_{i}^{2} / 2 \sigma_{m}^{2}}
$$

representing the zero mean Gaussian component, which corresponds to the state $m$ with the variance $\sigma_{m}{ }^{2}$.

It is also observed that, in this peaky, heavy-tailed marginal distribution, only a few coefficients have large values at positions of image singularities such as edges, while most others have very small values. Therefore, it is reasonable to simplify the multistate Gaussian mixture into a two-state representation as shown in (4). One state is used to describe large coefficient distribution and the other state is used to describe small coefficient distribution, i.e.,

$$
\begin{aligned}
p\left(w_{i}\right) & =P_{s} \cdot g\left(w_{i}, 0, \sigma_{s}^{2}\right)+P_{l} \cdot g\left(w_{i}, 0, \sigma_{l}^{2}\right) \\
g\left(w_{i}, 0, \sigma^{2}\right) & =\frac{1}{\sqrt{2 \pi} \sigma} e^{-w_{i}^{2} / 2 \sigma^{2}} \\
P_{s}+P_{l} & =1
\end{aligned}
$$

where the state of small coefficients is represented by subscript " $s$ " and the state of large coefficients by subscript " $l$." The a priori probabilities of the two states are represented by $P_{s}$ and $P_{l}$, respectively. The zero mean Gaussian component $g\left(w_{i}, 0, \sigma_{s}{ }^{2}\right)$ corresponding to the small state has a relatively small variance $\sigma_{s}{ }^{2}$, capturing the peakiness around zero (small coefficients), while the component $g\left(w_{i}, 0, \sigma_{l}^{2}\right)$ corresponding to the large state has a relatively large variance $\sigma_{l}^{2}$, capturing the heavy tails (large coefficients).

In order to model an image using the GMM, the model parameters $\left[P_{s}, P_{l}, \sigma_{s}^{2}, \sigma_{l}^{2}\right]$ need to be calculated and derived. Using the image coefficients as the training data, the GMM parameters can be obtained through an EM algorithm. The EM algorithm consists of two steps. The E step calculates the individual state probabilities for each wavelet coefficient $P_{s, i}, P_{l, i}$, and the $M$ step involves simple closed-form updates for the variances $\left[\sigma_{s}{ }^{2}, \sigma_{l}{ }^{2}\right]$ and the overall state probabilities $\left[P_{s}, P_{l}\right]$. The two steps interact with each other in an iterative process to help obtain a set of final converged GMM parameters, summarized as follows. 


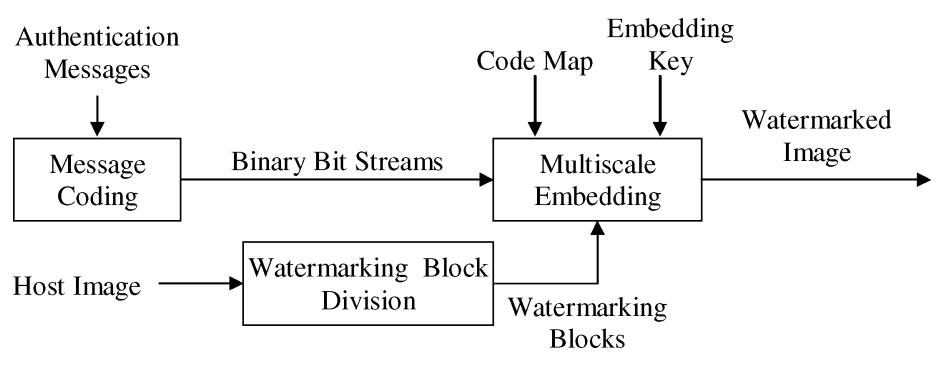

Fig. 1. Multiscale embedding of authentication messages.

\section{EM Algorithm for the GMM}

\section{Step 1) Initialization:}

Select an initial model estimate

$$
\theta(0)=\left[P_{s}(0), P_{l}(0), \sigma_{s}^{2}(0), \sigma_{l}^{2}(0)\right]
$$

where $\theta$ represents the GMM parameter set $\left[P_{s}, P_{l}, \sigma_{s}{ }^{2}, \sigma_{l}{ }^{2}\right]$.

Set iteration counter $n=0$.

Step 2) E step: Calculate the state probabilities for each wavelet coefficient $w_{i}$

$$
\begin{aligned}
P_{s, i}= & \frac{P_{s}(n) \cdot g\left(w_{i}, 0, \sigma_{s}^{2}(n)\right)}{P_{s}(n) \cdot g\left(w_{i}, 0, \sigma_{s}^{2}(n)\right)+P_{l}(n) \cdot g\left(w_{i}, 0, \sigma_{l}^{2}(n)\right)} \\
& i=1, \ldots, K \\
P_{l, i}= & \frac{P_{l}(n) \cdot g\left(w_{i}, 0, \sigma_{l}^{2}(n)\right)}{P_{s}(n) \cdot g\left(w_{i}, 0, \sigma_{s}^{2}(n)\right)+P_{l}(n) \cdot g\left(w_{i}, 0, \sigma_{l}^{2}(n)\right)} \\
& i=1, \ldots, K
\end{aligned}
$$

where $K$ represents the total number of coefficients in the wavelet subspace.

Step 3) M step: Update the model parameters to maximize the overall probabilities

$$
\left\{\begin{array}{c}
\theta(n) \rightarrow \theta(n+1) \\
\Downarrow \\
P_{s}(n+1)=\frac{1}{K} \sum_{i=1}^{K} P_{s, i} \\
P_{l}(n+1)=\frac{1}{K} \sum_{i=1}^{K} P_{l, i} \\
\sigma_{s}^{2}(n+1)=\frac{\sum_{i=1}^{K} w_{i}{ }^{2} \cdot P_{s, i}}{K \cdot P_{s}(n+1)} \\
\sigma_{l}{ }^{2}(n+1)=\frac{\sum_{i=1}^{K} w_{i}{ }^{2} \cdot P_{l, i}}{K \cdot P_{l}(n+1)} .
\end{array}\right.
$$

Step 4) Set $n=n+1$. If converged, then stop; otherwise, return to $\mathbf{E}$ step.

\section{GMM AND SECRET KEY-BASED \\ FRAGILE WATERMARKING}

The idea of the presented new fragile watermarking method is to embed watermark information into GMM parameters in a deterministic fashion for image integrity protection. As known, the DWT decomposes an image into multiscale subspaces. In the new fragile watermarking scheme, any wavelet subspace where we want to add watermark is divided into watermark blocks. The GMM and related EM algorithm are applied to these blocks to obtain their model parameters. Inspired by the methods in [9], [10], where the relationship of the selected DCT blocks is used to carry the image signature or the watermark message, our new method focuses on the large variance parameters in the GMMs of these watermark blocks in the wavelet domain and manages to adjust them to form special relationships through the modifications of the coefficients in the watermark blocks. Once these special relationships are formed, unauthorized image tampering or attacks imposed on the watermarked image will break these relationships and, hence, can be detected and localized. For security concerns, the formed authentication relationships are implemented based on a secret embedding key and code map so that it will be virtually impossible for an attacker to reproduce such relationships even if they know the algorithm details.

Fig. 1 has an overview of the watermark embedding process. Authentication messages are first translated into binary bit streams. Then the wavelet subspaces at multiple scales are divided into a number of wavelet watermarking blocks depending on the number of message bits being embedded and the number of wavelet scales these bits will spread into. The binary bit streams are then embedded into the wavelet watermarking blocks by forming some special relationships defined by the code map. The whole watermarking embedding process is secure and robust to malicious attacks because it is performed on a private key basis that guides a secret mapping between embedded bits and their corresponding wavelet watermarking blocks.

\section{A. Embedding Information Into the Statistical Model}

It is known that the 2-D DWT decomposes an image into three wavelet subspaces (horizontal, vertical and diagonal) at each scale. If the GMM and the EM algorithm are applied to these three subspaces, three different sets of model parameters will be obtained. We can modify the large coefficients of a single wavelet subspace so that its large variance parameter $\sigma_{l}^{2}$ will have the same value as that of another wavelet subspace $\sigma_{l}^{\prime 2}$. This specially formed relationship serves as the basis of the 
proposed fragile watermarking method. Any image operations or malicious attacks will inevitably change the wavelet coefficients; therefore, they will break this relationship and be detected. The modification of only large coefficients brings two advantages. First, the large coefficients usually represent singularities, such as image texture edges, in the spatial domain. The modifications on these large coefficients are relatively imperceptible by human vision. Second, large coefficients are not so many in a wavelet subspace; thus, the modifications made on them will not introduce much image distortion.

To make the large variance parameter $\sigma_{l}^{2}$ the same value as ${\sigma_{l}^{\prime}}^{2}$, each large coefficient $w_{i}$ will be modified by a certain amount $\Delta w_{i}$, such that

$$
\frac{1}{K} \sum_{i=1}^{P}\left[\left(w_{i}+\Delta w_{i}\right)^{2}-w_{i}^{2}\right]={\sigma_{l}^{\prime}}^{2}-\sigma_{l}^{2}
$$

where $P$ is the number of coefficients that are modified and $K$ is the total number of coefficients in the wavelet subspace. It is straightforward to see that once (9) is satisfied, the variance differences contributed by each coefficient modification will compensate the overall variance parameter difference.

Since the modifications of large coefficients, $w_{i}$, are independent from one another, there are numerous solutions satisfying (9). In the following Theorem 1, the optimal modification method that leads to the minimum image distortion is presented.

Theorem 1: Suppose $\sigma_{l}^{2}$ and ${\sigma_{l}^{\prime}}_{l}^{2}$ are the large variance parameters of two sets of the wavelet coefficients, denoted by $\mathcal{W}$ and $\mathcal{W}^{\prime}$. Let $w_{i}, i=1, \ldots, P$, represent the $P$ coefficients to be modified in the set $\mathcal{W}$ with $\sigma_{l}^{2}$, and the total number of coefficients in that wavelet subspace is $K$. If each coefficient, $w_{i}$, $i=1, \ldots, P$, is modified by a respective amount $\Delta w_{i}$ in order to make $\sigma_{l}^{2}$ and ${\sigma_{l}^{\prime}}^{2}$ equal, then the optimal way of modification with least image mean square distortion is that all coefficient $w_{i}$ are modified with a constant proportional rate $\alpha$, that is, $\Delta w_{i}=\alpha w_{i}, i=1, \ldots, P$, where the constant $\alpha$ is determined by the following equation:

$$
\sum_{i=1}^{P}\left\{\left[w_{i}(1+\alpha)\right]^{2}-w_{i}^{2}\right\}=K\left(\sigma_{l}^{\prime 2}-\sigma_{l}^{2}\right)
$$

Proof: The image mean square distortion after modification is

$$
J\left(\Delta w_{i} \mid i=1, \ldots, P\right)=\sum_{i=1}^{P} \Delta w_{i}^{2}
$$

To leverage the difference between the large variance parameters $\sigma_{l}^{2}$ and ${\sigma_{l}^{\prime}}^{2},(9)$ becomes a constraint condition

$$
\begin{aligned}
& D(\left.\Delta w_{i} \mid i=1, \ldots, P\right) \\
& \quad=\sum_{i=1}^{P}\left[\left(w_{i}+\Delta w_{i}\right)^{2}-w_{i}^{2}\right]-K\left({\sigma_{l}^{\prime}}^{2}-\sigma_{l}^{2}\right)=0 .
\end{aligned}
$$

The objective is to minimize the image distortion (11) subject to (12). Using the Lagrangian approach, we construct a Lagrangian relaxation function as follows:

$$
\begin{aligned}
& J^{\prime}\left(\Delta w_{i} \mid i=1, \ldots, P\right) \\
& =J\left(\Delta w_{i} \mid i=1, \ldots, P\right)+\lambda D\left(\Delta w_{i} \mid i=1, \ldots, P\right) \\
& =\sum_{i=1}^{P}\left[(1+\lambda) \Delta w_{i}^{2}+2 \lambda w_{i} \Delta w_{i}\right]-\lambda K\left({\sigma_{l}^{\prime}}^{2}-\sigma_{l}^{2}\right) .
\end{aligned}
$$

A minimization of the Lagrangian relaxation yields

$$
\frac{\partial J^{\prime}\left(\Delta w_{i} \mid i=1, \ldots, P\right)}{\partial\left(\Delta w_{i}\right)}=0, i=1, \ldots, P .
$$

Thus

$$
\Delta w_{i}=\frac{-\lambda w_{i}}{1+\lambda}=\alpha w_{i}, i=1, \ldots, P
$$

with

$$
\alpha=\frac{-\lambda}{1+\lambda} .
$$

That is to say, when all large coefficients $w_{i}$ are modified with a same constant proportional rate $\alpha$, it achieves the least image mean square distortion. Substituting the conclusion of (15) into (12), (10) is obtained to determine the constant $\alpha$.

Equation (10) is a quadratic function with respect to the proportional rate $\alpha$. Therefore, the value of $\alpha$ can be easily calculated.

It is noted that the two large variance parameters $\sigma_{l}^{2}$ and ${\sigma_{l}^{\prime}}^{2}$ should be obtained through the EM algorithm. In the modification by (12) and (15), the gap between $\sigma_{l}^{2}$ and ${\sigma_{l}^{\prime}}^{2}$ is compensated by a statistical approach other than the EM algorithm. In fact, after the modification by (15), there is still a small discrepancy between the updated parameter of $\sigma_{l}^{2}$ and the target parameter $\sigma_{l}^{\prime 2}$, calculated by the EM algorithm. Therefore, an iterative approach involving the modification and the EM algorithm in each single step is required to finally adjust the large variance parameter $\sigma_{l}^{2}$ to the target value ${\sigma_{l}^{\prime}}_{l}^{2}$, as demonstrated in Fig. 2.

\section{B. Secret Embedding Key and Code Map Based Multiscale Fragile Watermarking}

The formed special relationships can be used to implement and verify the existence of fragile watermarks. In order to embed fragile watermarks with sensitive information (such as a personal signature or logo) into the host image, a technique that involves proper watermarking block division to accommodate the embedded information bits is required, as shown in Fig. 1. Moreover, for secure embedding of watermark information, a secret embedding key and code map, which are unknown to potential attackers, are developed for the presented new watermarking method. 


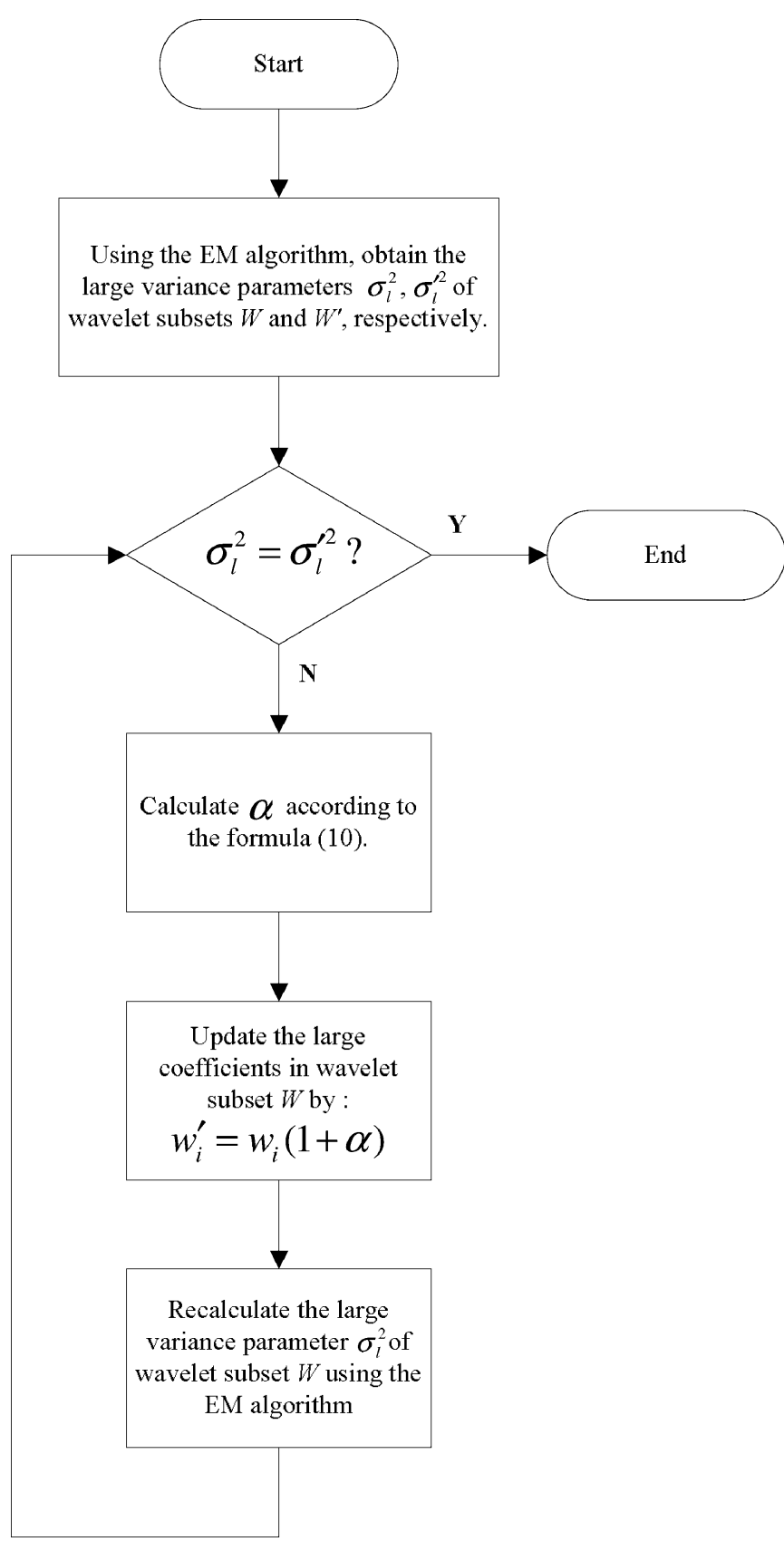

Fig. 2. Iterative approach for coefficient modification.

In order to embed a binary bit stream, instead of a single bit, into the wavelet subspace, a wavelet subspace is divided into $N \times N$ watermarking blocks and the value of $N$ depends on the number of information bits to be embedded. A simple and preliminary way to embed the bit stream is to use every three corresponding wavelet watermarking blocks obtained at the same position from the wavelet subspaces (horizontal, vertical, diagonal) at the same scale to form a special relationship to encode two message bits. A sample code map is shown in Table I, and it is not difficult to conceive other types of code maps.

In Table I, the parameters $\sigma_{l, H}^{2}, \sigma_{l, V}^{2}, \sigma_{l, D}^{2}$ represent the variances of the large coefficients of the three wavelet watermarking blocks obtained from horizontal subspace, vertical subspace and
TABLE I

SAMPLE Code MAP FOR MesSAGe Bits EMBEDding

\begin{tabular}{|c|c|}
\hline Formed Relationship & Coded Bits \\
\hline$\sigma_{l, V}^{2}=\sigma_{l, H}^{2}$ & 00 \\
\hline$\sigma_{l, V}^{2}=\sigma_{l, D}^{2}$ & 01 \\
\hline$\sigma_{l, H}^{2}=\sigma_{l, D}^{2}$ & 10 \\
\hline$\sigma_{l, V}^{2}=\sigma_{l, H}^{2}=\sigma_{l, D}^{2}$ & 11 \\
\hline
\end{tabular}

diagonal subspace, respectively. Various parameter equity relationships among $\sigma_{l, H}^{2}, \sigma_{l, V}^{2}$, and $\sigma_{l, D}^{2}$ can be formed to encode different two bits into these three wavelet watermarking blocks. Since there are $N^{2}$ groups of such wavelet watermarking blocks, at most $2 N^{2}$, message bits can be embedded at a single wavelet scale. Any unauthorized changes made in a specific area of the watermarked image will destroy the corresponding relationship and message bits; therefore, the tampering can be detected and localized. As the coded bits for each relationship are defined in the code map so that the same code map is required at the decoding end for proper watermark extraction. Without the knowledge of the code map, watermarks cannot be properly resolved by any potential attackers.

In many commercial applications, there are great security concerns that the preliminary message bits embedding scheme will be vulnerable to watermark counterfeiting if the watermarking implementation algorithm is known to malicious attackers. Since the presented watermarking algorithm employs a complicated iterative EM algorithm for variance computation and updating, certain algorithm parameters, such as initial model parameters and the convergence criteria, etc., will certainly affect the final convergence results. Note that, since the statistical properties of different watermark blocks are generally very similar, a subtle discrepancy will complete destroy the intended special relationship. Therefore, by keeping only a few algorithm parameters secret, certain security can still be warranted even if the general algorithm is known.

In addition, the security can be enhanced by incorporating a private embedding key to code the map associating the embedded bits to the selected wavelet watermarking blocks. The wavelet watermarking blocks are not necessarily obtained from the same positions, neither should the embedded bits follow the same order in their bit stream. Instead, the private key controls the embedding strategy and decides the positions of three wavelet watermarking blocks and their relationships to the pair of message bits to be embedded. Therefore, a malicious attacker has no way to manipulate the embedded watermarks even when $\mathrm{s} /$ he is fully aware of the entire watermarking techniques and procedures.

An example of using the secret embedding key is shown in Fig. 3, in which the group of three wavelet watermarking blocks with light shade is embedded with information bits 00 and the group with heavy shade is embedded with another two bits information 01 from the message bit stream. The secret embedding key itself can also be encoded into a binary bit stream that controls the wavelet watermarking block selection for each group to embed two bits information. To unambiguously define the three wavelet watermarking block positions in a group, a straightforward way is to use the wavelet subspace index and the wavelet 


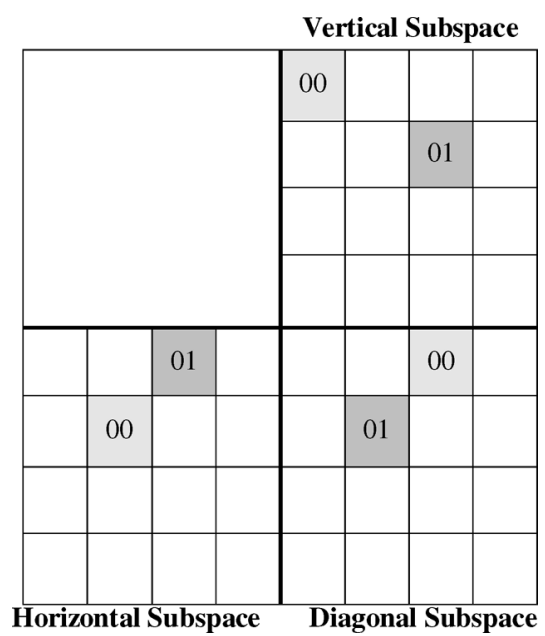

Fig. 3. Example of secret key based watermark embedding.

watermarking block coordinates within that subspace. It can be described as a format $\mathrm{W}-\mathrm{A}-\mathrm{B}$, where $\mathrm{W}$ is the wavelet subspace index, with a value ranging from 0 to 2 to represent the three different wavelet subspaces (vertical, horizontal, and diagonal), respectively, and $\mathrm{A}$ and $\mathrm{B}$ are the row and column indices of the wavelet watermarking blocks within that wavelet subspace, respectively, taking a value ranging from 0 to $N-1$, depending on the number of wavelet watermarking blocks necessary. Symbols $\mathrm{W}, \mathrm{A}$, and $\mathrm{B}$ are all binary encoded in real implementations. Applying the above protocol, the three light shaded blocks in Fig. 3 can be coded as 00-00-00, 01-01-01, and 10-00-10, respectively. Therefore, the group that contains these three blocks to embed bits 00 is coded as 00-00-00-01-01-01-10-00-10. Once the secrete embedding key is generated, it is also possible to encrypt and embed the key, code map and other secret algorithm parameters, into the host image as a robust watermark so that the decoder does not need any information other than a prechosen encryption key and the image itself for watermark extraction.

The new fragile watermarking method can be applied to multiple wavelet scales to increase the embedding rate. Furthermore, by embedding watermarks to multiple wavelet scales, we are able to distinguish some normal image operations such as JPEG compression from malicious attacks, which is a desired property in some semi-fragile applications. As will be shown in the simulations, the compression has a gradual decreasing pattern on the distortion of fragile watermarks as the wavelet scale increases. Malicious attacks do not have this pattern.

\section{Discussion of the Security}

Recent literature on robust watermarking security [13], [14] have indicated that security refers to "the inability by unauthorized users to access the watermarking channel," and such an access refers to "remove, detect and estimate, write and modify the raw watermarking bits." Using the same concept, in fragile watermarking, we may define the security as the inability by unauthorized users to manipulate the watermarked authentic image without being detected, and such manipulation refers to detect, estimate and modify the watermarking bits.
The security of the presented secure fragile watermarking method depends on the secrecy of three components: 1) the algorithm parameters: the initial model parameters and convergence criteria used in EM algorithm, 2) the embedding key and code map, and 3) a possible secret encryption key to encrypt components 1) and 2).

According to [13], the security is evaluated by two criteria: the security level and the security work. Security level refers to the number of observations needed to disclose the watermarking secrets, while the security work refers to the complexity of the algorithm that attackers use to extract the watermarking secrets from observations. In the security analysis of our method, we assume the attackers already know every detail of the watermarking method, including the employed statistical model, the EM algorithm, and the watermarking procedures, except the above-mentioned three secret components. If the attackers do not know the initial model parameters and the convergence criteria used in the EM algorithm during watermarking, they are not able to find the secrete key and code map to regenerate the formed relationship (used to carry watermarking bits) from the watermarked image. The exhaustive search for the secret initial parameters and convergence criteria is sophisticate and computationally expensive since the search space is continuous. Therefore, the security work is expected to be huge and very complicated. Without proper knowledge of the secret information, the attackers are hardly able to estimate and further modify the watermarked information.

On the other hand, note that the first two secrete components can be arbitrarily generated each time and they can be encrypted and embedded into the image using a robust watermark. Therefore, in such case, the break of one set of the secret parameters and embedding map does not jeopardize the security of other watermarked images. The general security level of the presented method will then rely on the security level of the encryption, which is independent of the watermarking parameters, if multiple observations are available to attackers. The security level of the encryption depends on the specific encryption method (such as RSA or DES) and its analysis is beyond the scope of our paper.

Security is an important and involved aspect of watermarking. The above discussion is preliminary and conceptual. The quantitative analysis of security will have to depend on the specific implementation of the system and the analysis of the possible attacks. The full in-depth analysis of the security of the specific algorithm implementation for applications will be another important and involved research topic.

\section{Embedding Payload}

Assume the host image size is $N \times N$ and the watermarking block size is $B \times B$ for all wavelet scales. Let $i=1, \ldots, K$, represent the $K$ wavelet scales that are used to embed watermarks. The embedding payload $C$ of the proposed watermarking scheme can be formulated as follows:

$$
C=\sum_{i=1}^{K} 2\left(\frac{N}{2^{i} B}\right)^{2} .
$$


With the decrease of the block size $B$, the embedding payload will increase but the watermarking distortion will increase as well at the same time. Moreover, an extremely small block size chosen will lead to insufficient training data for the statistical model and reduce the robustness and accuracy of the derived model parameters, hence impairing the effectiveness of the watermarking method based on the statistical modeling. Researchers doing image retrieval have indicated that for an accurate and robust estimation of statistical model parameters in the wavelet domain, the smallest wavelet block used for training should not be less than $16 \times 16$ [15], [16], which is consistent and agreed with our empirical observations. On the other hand, since watermarking at low-frequency wavelet scales will incur substantial image distortions, at most, four levels of wavelet decomposition are selected for watermark embedding. Therefore, an empirical embedding payload can be obtained from (17) and expressed as

$$
C=\sum_{i=1}^{4} 2\left(\frac{N}{2^{i} \cdot 16}\right)^{2}=\frac{1}{128} \sum_{i=1}^{4}\left(\frac{N}{2^{i}}\right)^{2} .
$$

It is obvious that, for a $512 \times 512$ image, as many as 512 watermark bits can be embedded at the finest wavelet scale and total 680 bits in all four wavelet scales.

\section{EXPERIMENTAL RESULTS}

The $512 \times 512$ images of "Peppers" and "Lena" are used to demonstrate the effectiveness of the new fragile watermarking method. A watermark is first embedded into the "Peppers" image based on a preliminary embedding scheme and then embedded into the Lena image based on a secret embedding key. Later, a 128-bit signature is embedded into the Lena image. In the first two cases, our lab logo "CASPAL" is used as the fragile watermarks to be embedded. Since a 5 bit stream is used to encode the 26 alphabets ( 00001 for A, 00010 for B, and so on), the total number of bits required to embed the logo is 30 . According to Table I, at least 15 blocks are needed for each embedding wavelet subspace. To facilitate the operation, we divide the wavelet subspace into $16(4 \times 4)$ blocks so that 32 message bits (representing the logo) are actually embedded.

Fig. 4 shows the wavelet subspaces that contain 32 embedded bits of "CASPAL" in the "Peppers" image. Every three wavelet watermarking blocks obtained at the same position from the wavelet subspaces are used to embed two message bits. For example, the three shaded wavelet watermarking blocks embed message bits "00," which are the initial bits of the letter "C," using the relationship shown in Table I.

Figs. 5 and 6 display the original image and the watermarked "Peppers" images, respectively. As can be seen, embedding the lab logo does not cause any perceptible distortion on the watermarked image.

An advantage of the presented watermarking method is that it modifies few image data and has little image distortion. Figs. 7 and 8 plot the wavelet coefficients of the original and the watermarked "Peppers" images, respectively. The changes made on the wavelet coefficients can be observed by comparing the two plots. It is calculated that the total number

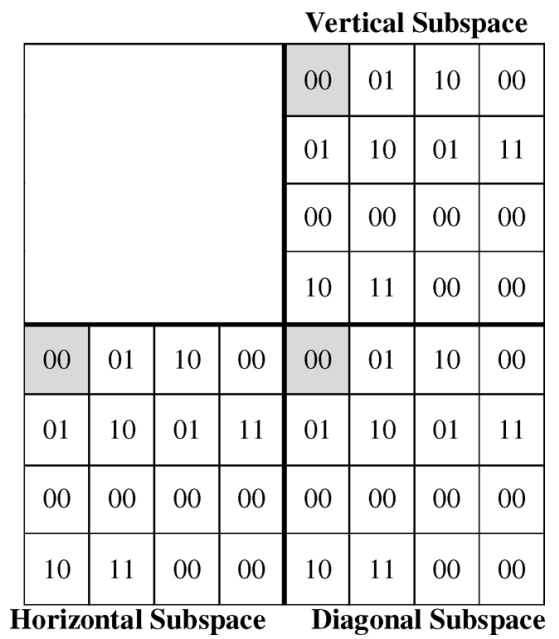

Fig. 4. Message bits embedded into the wavelet watermarking blocks.

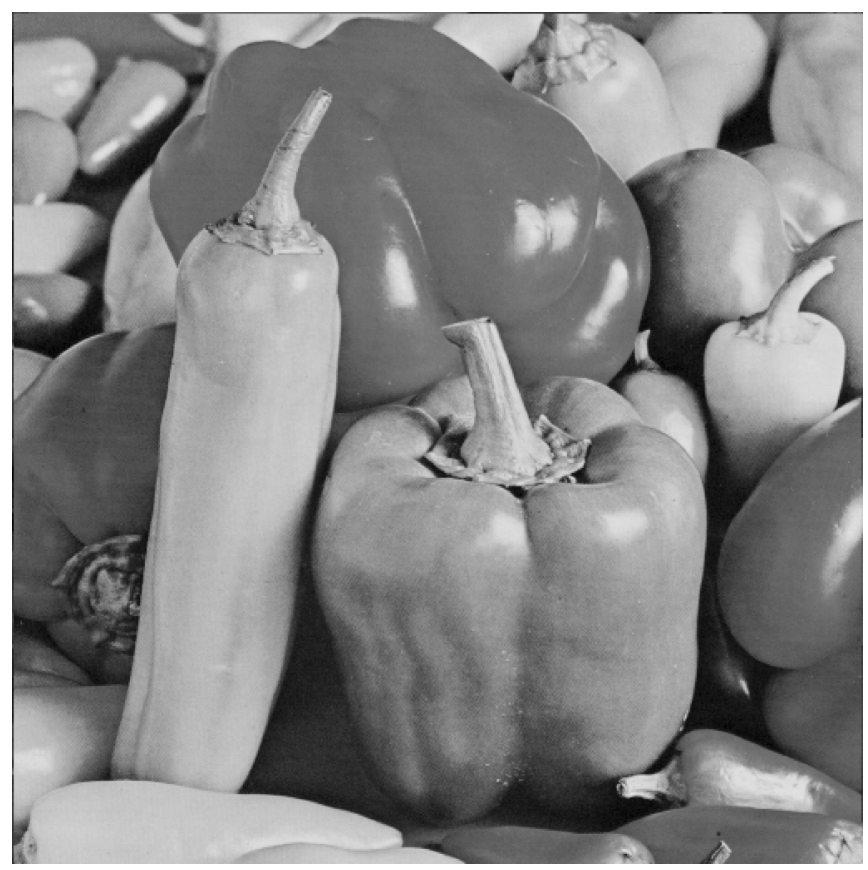

Fig. 5. Original "Peppers" image before watermarking.

of modified coefficients in Fig. 8 is 680 (out of $512 \times 512$ image data). Compared with some conventional fragile watermarking methods [3], [5], [6] that modify nearly half of the image pixels, the statistical model based approach modifies much fewer image data. Besides, the changes are only made on large coefficients that represent image edges in the space domain. When watermarks are embedded at image edges, they are generally more imperceptible by human vision, as shown in Fig. 6. Taking watermarks as a noise signal introduced in the host image, as an indicator of the image distortion caused by watermarking, the PSNR (peak-signal-to-noise-ratio) is calculated in the experiments. The PSNR value is $52.12 \mathrm{~dB}$ for Fig. 6, indicating very little image watermarking distortion on the original host image.

To test the sensitivity of image tampering detection, first a single pixel tampering experiment is performed 20 times. Each 


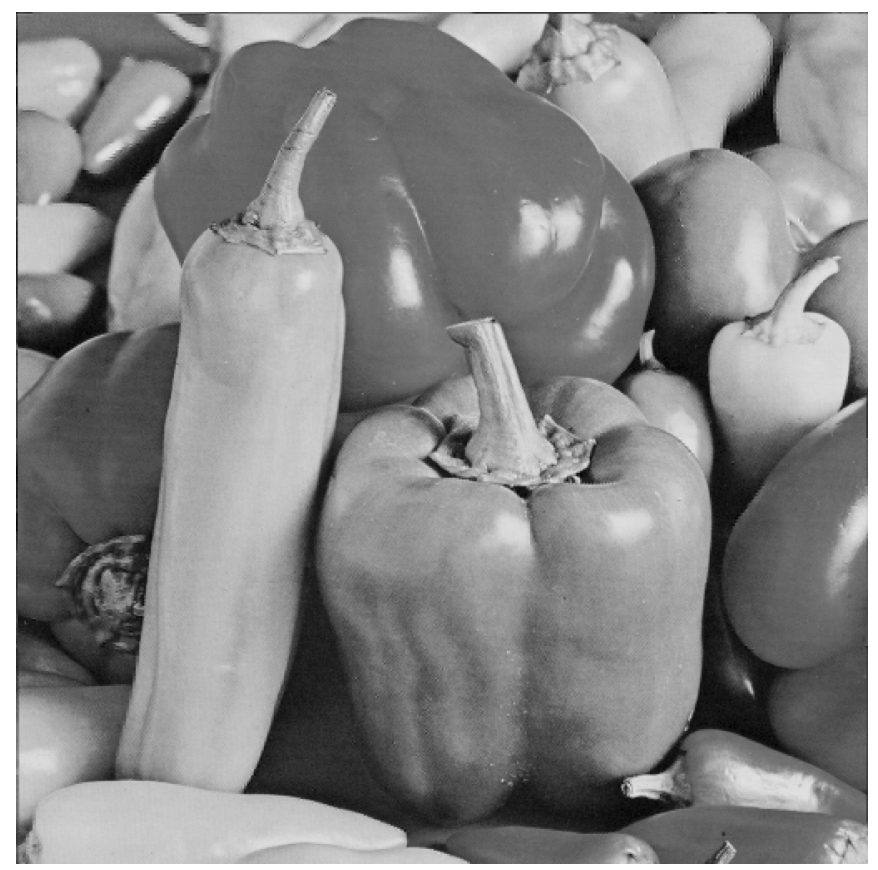

Fig. 6. Watermarked "Peppers" image with the lab logo "CASPAL" embedded.

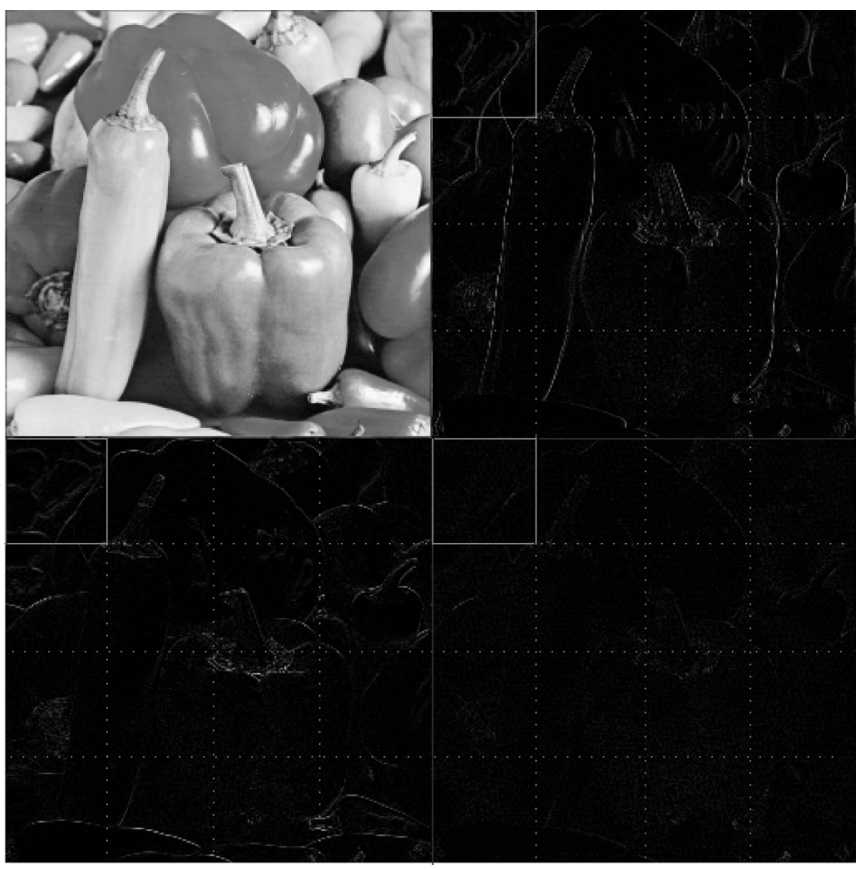

Fig. 7. DWT of the original "Peppers" image at wavelet scale 1.

time a randomly selected pixel is modified by a small amount (grayscale magnitude of 10) and its impact to the embedded watermark is recorded. Table II shows the mean value of relative parameter differences deviated from the constructed parameter equity relationship as in Table I, which can represent the sensitivity of the watermark to image tampering.

As can be seen, no matter how slight the tampering is, it will be detected because it destroys the formed parameter equity relationship by a noticeable amount. The location of the tampering can also be determined since it only destroys the message bits at the positions of the tampering.

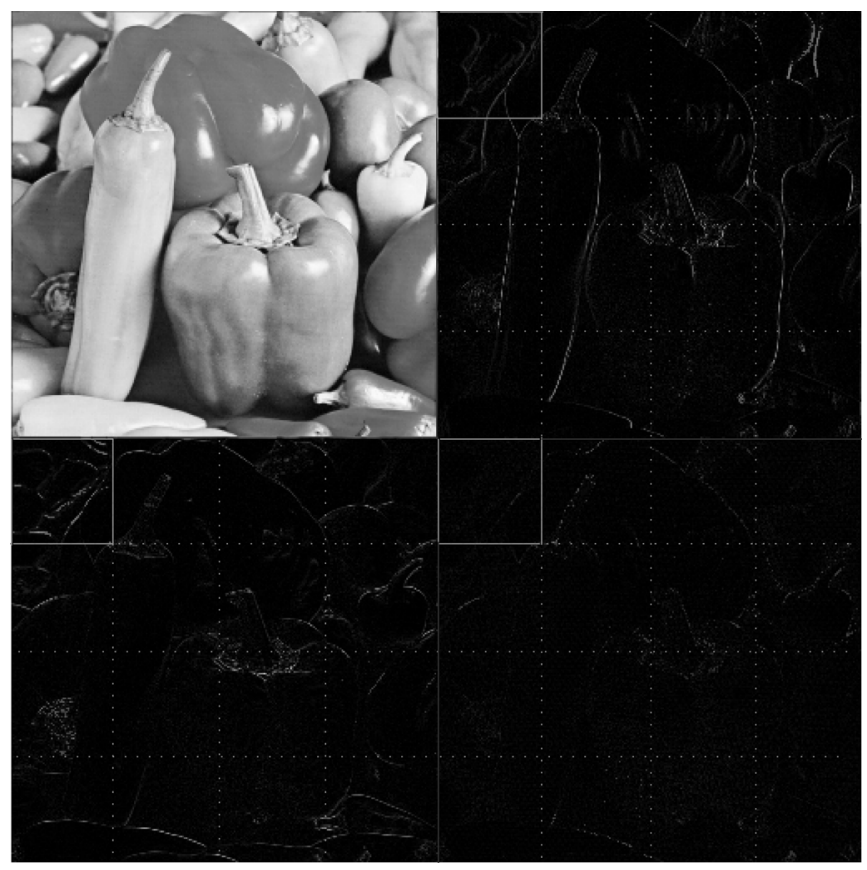

Fig. 8. DWT of the watermarked "Peppers" image with the lab logo "CASPAL" embedded at wavelet scale 1 .

\section{TABLE II}

Average Parameter Difference Caused by Single-PiXel MODIFICATION (FOR THE WATERMARKED "PEPPERS" IMAGE WITH THE LAB LOGO “CASPAL” EMBEDDED)

\begin{tabular}{|c|c|}
\hline Number of Trials & 20 \\
\hline Average Parameter Difference & $0.11 \%$ \\
\hline Corresponding Message Bits Destroyed & Yes \\
\hline
\end{tabular}

TABLE III

PARAMETER DifFerenCE CAUSED BY NOISE (FOR THE WATERMARKED "PepPeRs" IMAGE With the LAB LOGO “CASPAL” EMBEDDED)

\begin{tabular}{|l|c|c|c|c|c|}
\hline & \multicolumn{5}{|c|}{ Noise Variance } \\
\hline \multirow{2}{*}{$\begin{array}{l}\text { Scale } \\
\text { Level }\end{array}$} & & 0.0001 & 0.0002 & 0.0005 & 0.0010 \\
\cline { 2 - 6 } & 1 & $1.25 \%$ & $2.37 \%$ & $6.06 \%$ & $19.42 \%$ \\
\cline { 2 - 6 } & 2 & $4.65 \%$ & $8.59 \%$ & $10.27 \%$ & $17.49 \%$ \\
\hline
\end{tabular}

Table III lists the relative parameter differences caused by additive noise with different variances while the watermarks are embedded into two wavelet scales. As can be seen, no matter how slight the tampering is, the previously formed parameter equity relationship will be broken and the tampering will be detected. Moreover, the parameter differences tend to become larger with the increase of the tampering strength.

The presented fragile watermarking method has an advantage of distinguishing some normal image operations such as JPEG compression from malicious attacks when the watermarks are embedded into multiple wavelet scales. Table IV shows the impact of the JPEG compression on the watermarked image that has watermarks embedded at scales 1 to 4 . The numbers in Table IV represent relative parameter differences. It can be seen that at the same compression level, the relative parameter difference decreases as the wavelet scale increases. On the other hand, 
TABLE IV

PARAMETER DIFFERENCE CAUSED BY JPEG COMPRESSION (FOR THE WATERMARKED "PEPPERS" IMAGE WITH THE LAB LOGO “CASPAL” EMBEDDED)

\begin{tabular}{|l|c|c|c|c|c|}
\hline & \multicolumn{5}{|c|}{ Compression Ratio } \\
\hline \multirow{3}{*}{ Scale } & & $60 \%$ & $38 \%$ & $25 \%$ & $15 \%$ \\
\cline { 2 - 6 } \begin{tabular}{l} 
Level \\
\cline { 2 - 6 }
\end{tabular} & 1 & $1.47 \%$ & $2.36 \%$ & $2.86 \%$ & $4.53 \%$ \\
\cline { 2 - 6 } & 2 & $0.84 \%$ & $1.27 \%$ & $1.76 \%$ & $2.75 \%$ \\
\cline { 2 - 6 } & 3 & $0.21 \%$ & $0.31 \%$ & $1.18 \%$ & $1.99 \%$ \\
\hline
\end{tabular}

TABLE V

Parameter DifFerence CAused by SOME Malicious AtTacks (FOR THE WATERMARKED "PEPPERS" IMAGE WITH THE LAB LOGO “CASPAL” EMBEDDED)

\begin{tabular}{|l|c|c|c|}
\hline & \multicolumn{3}{|c|}{ Malicious Attacks } \\
\hline \multirow{3}{*}{ Scale } & & Gaussian White Noise & Content Change \\
\cline { 2 - 4 } Level & 1 & $3.06 \%$ & $1.65 \%$ \\
\cline { 2 - 4 } & 2 & $7.27 \%$ & $0.34 \%$ \\
\cline { 2 - 4 } & 3 & $6.13 \%$ & $0.98 \%$ \\
\cline { 2 - 4 } & 4 & $14.75 \%$ & $1.13 \%$ \\
\hline
\end{tabular}

\begin{tabular}{|c|c|c|c|c|c|c|c|}
\hline & & & & \multicolumn{4}{|c|}{ Vertical Subspace } \\
\hline & & & & 00 & 01 & 10 & 00 \\
\hline & & & & 01 & 10 & 01 & 11 \\
\hline & & & & 00 & 00 & 00 & 00 \\
\hline & & & & 10 & 11 & 00 & 00 \\
\hline 00 & 00 & 01 & 00 & 01 & 00 & 00 & 10 \\
\hline 11 & 00 & 00 & 11 & 00 & 01 & 00 & 00 \\
\hline 01 & 00 & 10 & 10 & 11 & 00 & 10 & 10 \\
\hline 10 & 01 & 00 & 00 & 00 & 11 & 01 & 00 \\
\hline
\end{tabular}

Fig. 9. Message bits embedded into the wavelet watermarking blocks based on a secret embedding key.

some malicious attacks, including additive Gaussian white noise and deliberate slight change of image contents (arbitrary small modifications of 100 random pixels), are simulated. The simulation results are shown in Table $\mathrm{V}$. The parameter changes due to these malicious attacks do not have the same characteristic as the image compression. Therefore, this feature can be used to distinguish image JPEG compression from these malicious attacks for semi-fragile watermarking applications.

In the second experiment, the same lab logo "CASPAL" is embedded into the $512 \times 512$ image of "Lena" depending on a secret embedding key. The wavelet watermarking block selection for embedding the 32 bits watermark is displayed in Fig. 9. Figs. 10 and 11 display the original and the watermarked "Lena" images, respectively. Again, it is observed that the proposed wa-

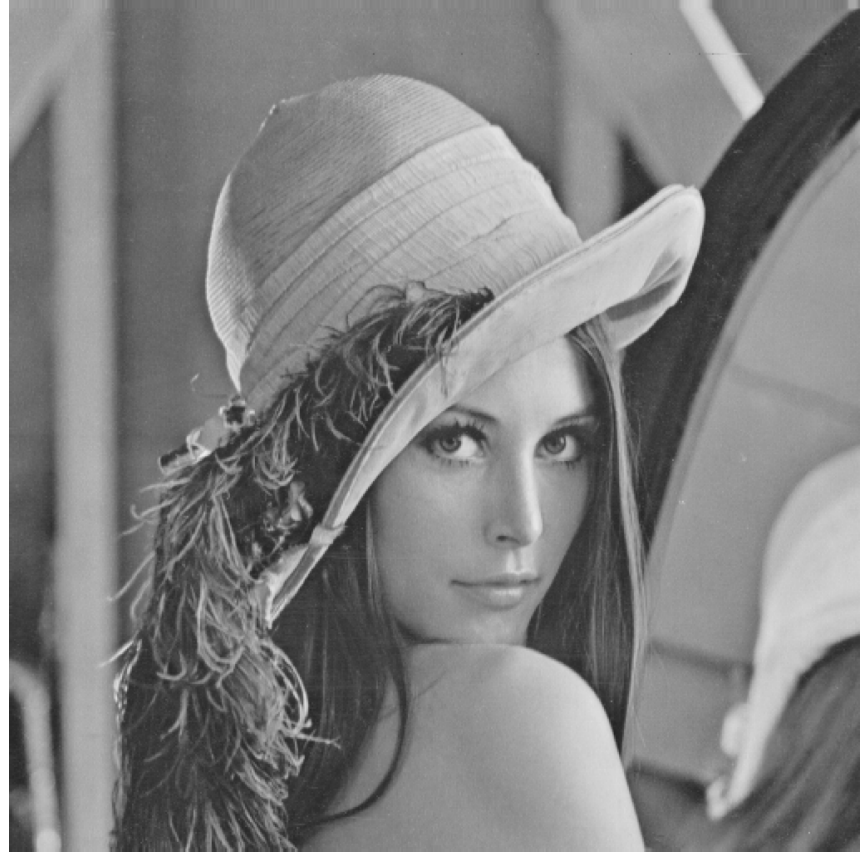

Fig. 10. Original "Lena" image before watermarking.

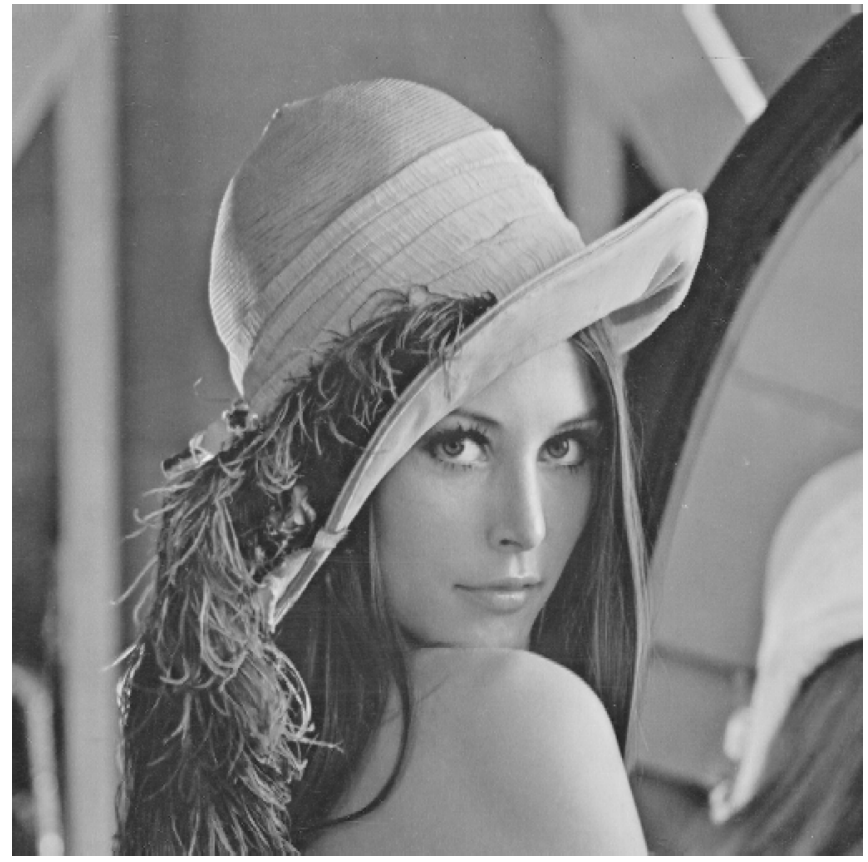

Fig. 11. Watermarked "Lena" image with the lab logo "CASPAL" embedded.

termarking method does not introduce any perceptible distortion on the host image through watermark embedding.

The high quality of the watermarked image is assured by the high PSNR value and fewer modified image data in watermarking. Figs. 12 and 13 plot the wavelet coefficients of the original and the watermarked "Lena" images, respectively. The total number of modified coefficients in Fig. 13 is only 852 and the PSNR value is $50.27 \mathrm{~dB}$, which indicates that little image distortion is introduced by watermarking.

To demonstrate the semi-fragile functionality of the presented method, some malicious attacks like the Gaussian white noise 


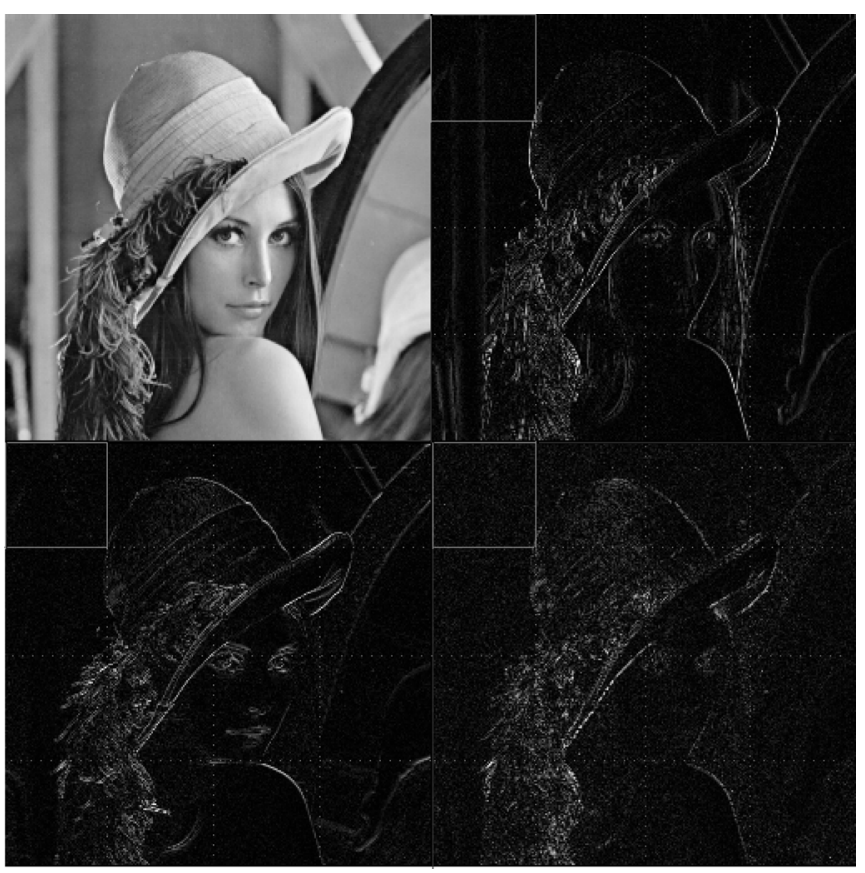

Fig. 12. DWT of the original "Lena" image at wavelet scale 1.

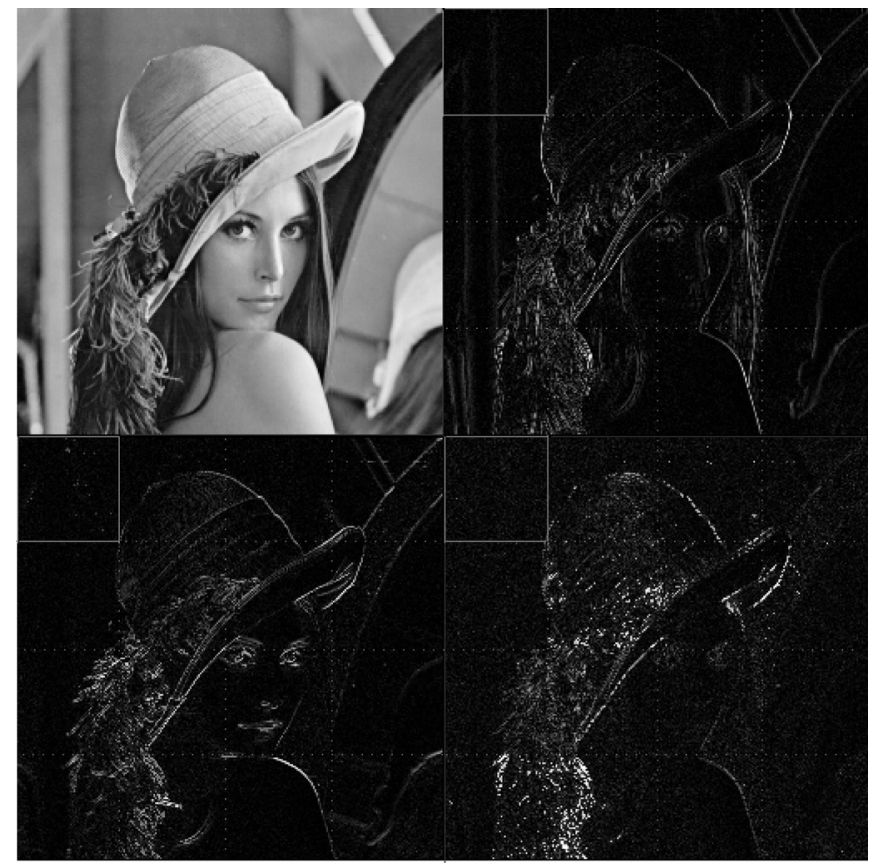

Fig. 13. DWT of the watermarked "Lena" image with the lab logo "CASPAL" embedded at wavelet scale 1 .

and image content tampering are imposed on the watermarked "Lena" image. Table VI lists the relative parameter differences caused by these malicious attacks at different wavelet scales, and Table VII displays the JPEG compression impact on the parameter differences at these scale levels. It can be observed from these two tables that the changes of the parameter differences from malicious attacks do not have the same characteristic as that from the JPEG compression. Therefore, we are able to distinguish image JPEG compression from the malicious attacks by spreading watermarks into multiple wavelet scales.
TABLE VI

Parameter Difference Caused by Some Malicious AtTaCKs (For the WATERMARKED "LENA" IMAGE With THE LAB LOGO “CASPAL" EMBEDDED)

\begin{tabular}{|l|c|c|c|}
\hline & \multicolumn{3}{|c|}{ Malicious Attacks } \\
\hline \multirow{3}{*}{\begin{tabular}{l} 
Scale \\
\multirow{2}{*}{ Level }
\end{tabular}} & & Gaussian White Noise & Content Change \\
\cline { 2 - 4 } & 1 & $8.35 \%$ & $4.78 \%$ \\
\cline { 2 - 4 } & 2 & $4.62 \%$ & $5.67 \%$ \\
\cline { 2 - 4 } & 3 & $5.52 \%$ & $1.73 \%$ \\
\hline
\end{tabular}

TABLE VII

PARAMETER DIFFERENCE CAUSED BY JPEG COMPRESSION (FOR THE WATERMARKED "LENA" IMAGE With THE LAB LOGO “CASPAL" EMBEDDED)

\begin{tabular}{|l|c|c|c|c|c|}
\hline & \multicolumn{5}{|c|}{ Compression Ratio } \\
\hline \multirow{3}{*}{ Scale } & & $60 \%$ & $38 \%$ & $25 \%$ & $15 \%$ \\
\cline { 2 - 6 } Level & 1 & $1.68 \%$ & $2.76 \%$ & $3.23 \%$ & $4.11 \%$ \\
\cline { 2 - 6 } & 2 & $0.75 \%$ & $1.23 \%$ & $1.62 \%$ & $2.52 \%$ \\
\cline { 2 - 6 } & 3 & $0.30 \%$ & $0.57 \%$ & $0.96 \%$ & $1.65 \%$ \\
\cline { 2 - 6 } & 4 & $0.17 \%$ & $0.34 \%$ & $0.60 \%$ & $1.04 \%$ \\
\hline
\end{tabular}

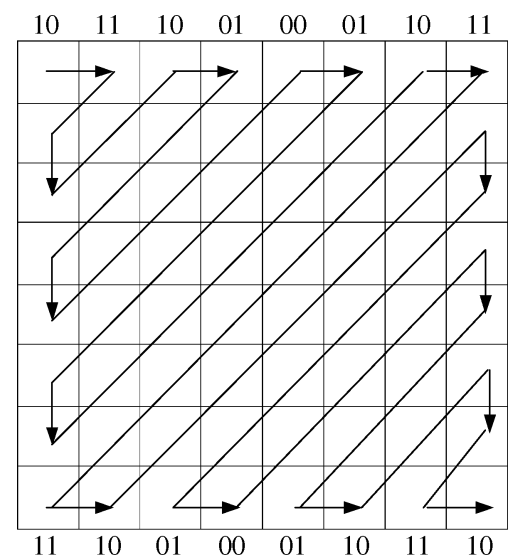

Fig. 14. The 128 bit-signature embedded into the wavelet watermarking blocks.

In the third experiment, a 128-bit signature with a pattern shown in Fig. 14 is embedded into the "Lena" image. It can be seen that the watermarked image shown in Fig. 15 has little distortion. The modification of wavelet coefficients can be observed visually through comparing the plotted wavelet subspaces of original "Lena" image and watermarked "Lena" image, as given by Figs. 16 and 17, respectively. The total number of modified coefficients is only 1077 and the PSNR value is $48.96 \mathrm{~dB}$, assuring high quality of the watermarked image when substantial number of bits are embedded.

By first embedding the 128-bit signature at the finest wavelet scale and then spreading it into subsequent scales, a semi-fragile watermark to distinguish the JPEG compression from malicious attacks can be achieved. Tables VIII and IX show the parameter differences incurred by the JPEG compression and some malicious attacks, respectively. The special property of the JPEG compression at multiple wavelet scales helps achieve the semifragile watermarking. 


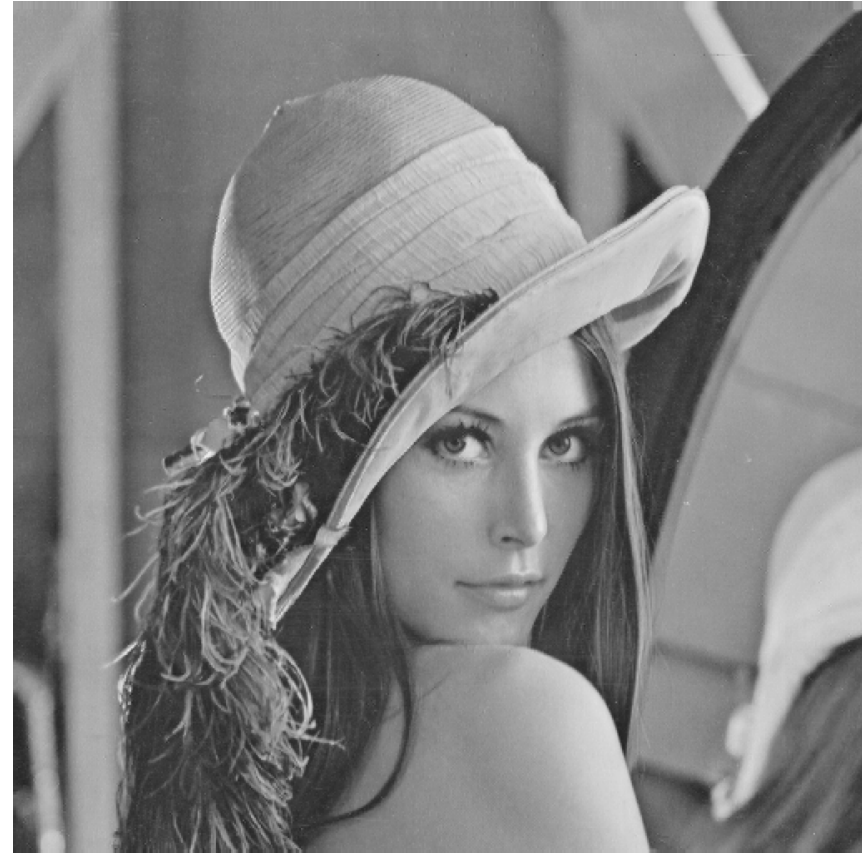

Fig. 15. Watermarked "Lena" image with a 128 bit signature embedded.

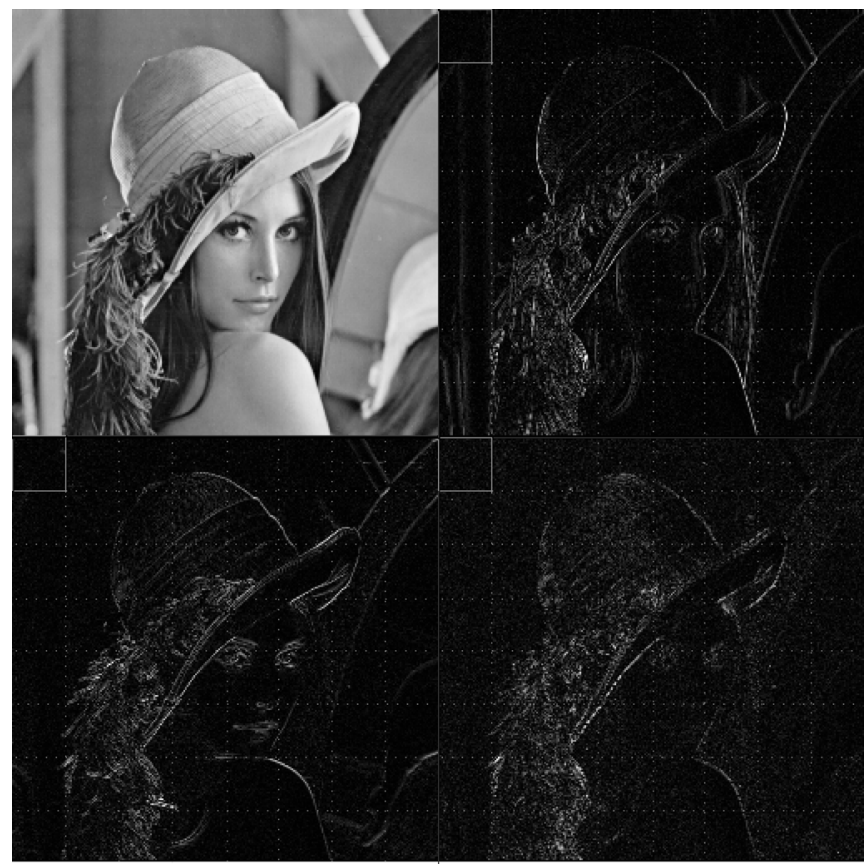

Fig. 16. DWT of the original "Lena" image at wavelet scale 1 with watermark block division for 128 bit signature embedding.

\section{CONCLUSION}

In this paper, a novel secure multiscale fragile watermarking method is presented. A Gaussian mixture statistical model is developed, and its model parameters are utilized to embed watermarks. The new method modifies only a very small amount of image data and has little image distortion for watermark embedding. The fragile watermarks can be securely embedded into a host image depending on the sophisticate GMM based embedding algorithm, a code map, and a proposed new secret

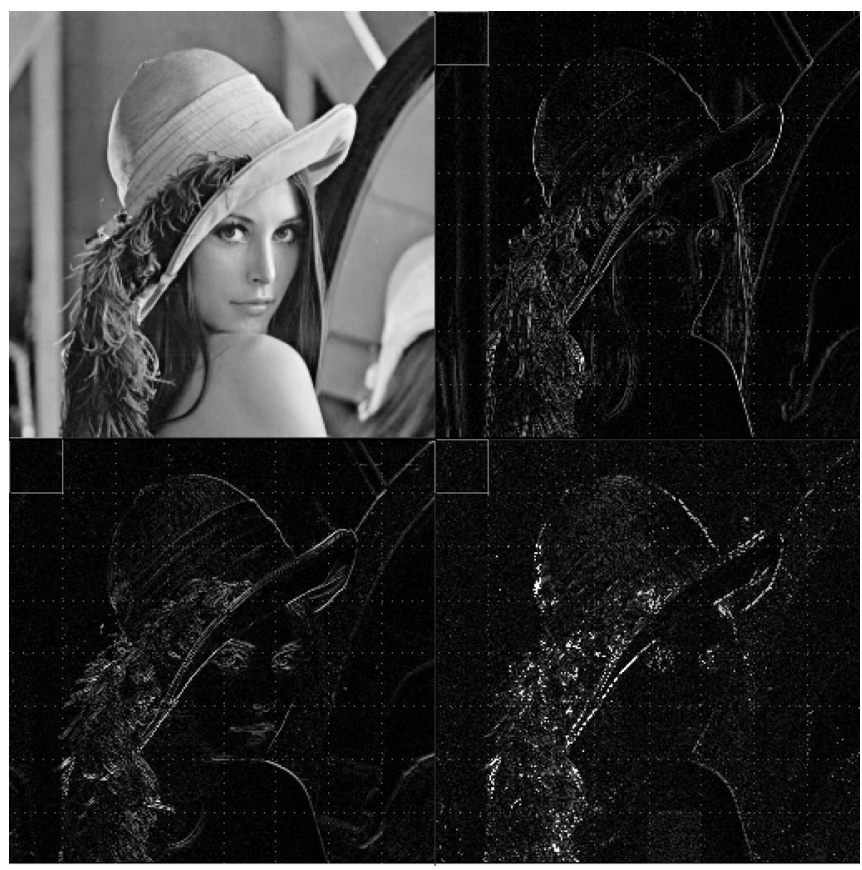

Fig. 17. DWT of the watermarked "Lena" image with a 128 bit signature embedded at wavelet scale 1 .

TABLE VIII

Parameter Difference Caused by Some Malicious AtTacks (For the WATERMARKED "LENA" IMAGE With A 128 Bit SignATURE EMBEDDED)

\begin{tabular}{|l|c|c|c|}
\hline & \multicolumn{3}{|c|}{ Malicious Attacks } \\
\hline \multirow{3}{*}{ Scale } & & Gaussian White Noise & Content Change \\
\cline { 2 - 4 } Level & 1 & $6.66 \%$ & $5.38 \%$ \\
\cline { 2 - 4 } & 2 & $8.48 \%$ & $3.59 \%$ \\
\cline { 2 - 4 } & 3 & $9.78 \%$ & $4.74 \%$ \\
\cline { 2 - 4 } & 4 & $4.32 \%$ & $2.49 \%$ \\
\hline
\end{tabular}

TABLE IX

PARAMETER DIFFERENCE CAUSED BY JPEG COMPRESSION (FOR THE WATERMARKED "LENA" IMAGE WITH A 128 BIT SigNATURE EMBEDDED)

\begin{tabular}{|l|c|c|c|c|c|}
\hline & \multicolumn{5}{|c|}{ Compression Ratio } \\
\hline \multirow{3}{*}{$\begin{array}{l}\text { Scale } \\
\text { Level }\end{array}$} & & $60 \%$ & $38 \%$ & $25 \%$ & $15 \%$ \\
\cline { 2 - 6 } & 1 & $2.10 \%$ & $3.27 \%$ & $3.81 \%$ & $5.04 \%$ \\
\cline { 2 - 6 } & 2 & $1.31 \%$ & $1.52 \%$ & $1.70 \%$ & $2.03 \%$ \\
\cline { 2 - 6 } & 3 & $0.81 \%$ & $0.83 \%$ & $1.19 \%$ & $1.47 \%$ \\
\cline { 2 - 6 } & 4 & $0.55 \%$ & $0.43 \%$ & $0.96 \%$ & $1.25 \%$ \\
\hline
\end{tabular}

embedding key scheme, which can also be encrypted and embedded into the same host image using robust watermarking approaches. The security of the new method is briefly analyzed and it is shown that the present fragile watermarking system is invulnerable to watermark counterfeiting because the secret algorithm parameters, the embedding key and code map, required at the decoding end for watermark extraction, are difficult to be broken by potential attackers. Any unauthorized changes that remove the embedded watermarks will be detected and localized. A semi-fragile watermarking approach can be achieved by applying the new method to multiple wavelet scales so that 
it can distinguish some normal image operations, such as JPEG compression, from malicious attacks.

\section{REFERENCES}

[1] B. B. Zhu, M. D. Swanson, and A. H. Tewfik, "When seeing isn't believing," IEEE Signal Proces. Mag., vol. 21, no. 2, pp. 40-49, Mar. 2004.

[2] M. U. Celik, G. Sharma, E. Saber, and A. M. Tekalp, "A hierarchical image authentication watermark with improved localization and security," in Proc. IEEE Int. Conf. Image Processing, Oct. 2001, vol. 2, pp. 502-505.

[3] S. Walton, "Information authentication for a slippery new age," $\mathrm{Dr}$. Dobbs J., vol. 20, no. 4, pp. 18-26, Apr. 1995.

[4] P. W. Wong, "A watermark for image integrity and ownership verification," in Proc. IS\&T PIC Conf., May 1998, pp. 374-379.

[5] — , "A public key watermark for image verification and authentication," in Proc. IEEE Int. Conf. Image Processing, Oct. 1998, vol. 1, pp. 445-449.

[6] A. Paquet and R. Ward, "Wavelet-based digital watermarking for image authentication," in Proc. IEEE Canad. Conf. Electrical and Computer Engineering, May 2002, vol. 2, pp. 879-884.

[7] J. Fridrich, "A hybrid watermark for tamper detection in digital images," in Proc. Int. Symp. Signal Processing and Applications, Aug. 1999, pp. 301-304.

[8] C. Y. Lin and S. F. Chang, "Semi-fragile watermarking for authenticating JPEG visual content," in Proc. SPIE Security and Watermarking of Multimedia Contents II, Jan. 2000, pp. 140-151.

[9] — "A robust image authentication method distinguishing JPEG compression from malicious manipulation," IEEE Trans. Circuits Syst. Video Technol., vol. 11, no. 2, pp. 153-168, Feb. 2001.

[10] E. Koch and J. Zhao, "Towards robust and hidden image copyright labeling," in Proc. IEEE Workshop on Nonlinear Signal and Image Processing, Jun. 1995, pp. 452-455.

[11] Q. B. Sun and S. F. Chang, "Semi-fragile image authentication using generic wavelet domain features and ECC," in Proc. IEEE Int. Conf. Image Processing, Sep. 2002, vol. 2, pp. 901-904.

[12] J. Romberg, H. Choi, and R. Baraniuk, "Bayesian tree-structured image modeling using wavelet-domain hidden Markov models," IEEE Trans. Image Process., vol. 10, no. 7, pp. 1056-1068, Jul. 2001.

[13] F. Cayre, C. Fontaine, and T. Furon, "Watermarking attack: Security of WSS techniques," in Proc. Int. Workshop on Digital Watermarking, Seoul, Korea, Oct. 2004, pp. 171-183.

[14] P. Comesaña, L. Pérez-Freire, and F. Pérez-González, "An information-theoretic framework for assessing security in practical watermarking and data hiding scenarios," presented at the Int. Workshop on Image Analysis for Multimedia Interactive Services Montreux, Switzerland, Apr. 2005.
[15] M. N. Do and M. Vetterli, "Wavelet-based texture retrieval using generalized Gaussian density and Kullback-Leibler distance," IEEE Trans. Image Process., vol. 11, no. 2, pp. 146-158, Feb. 2002.

[16] T. Chang and C.-C. J. Kuo, "Texture analysis and classification with tree-structured wavelet transform," IEEE Trans. Image Process., vol. 2, no. 4, pp. 429-441, Oct. 1993.

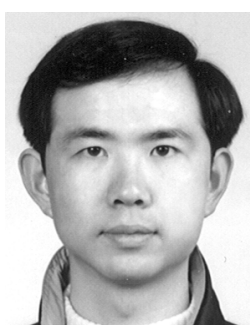

Hua Yuan was born in Shanghai, China, in 1974. He received the B.S. degree from Fudan University, China, and the M.A.Sc. degree from Ryerson University, Toronto, ON, Canada, in 1997 and 2004, respectively, both in electrical engineering. He is currently pursuing the Ph.D. degree.

From 1997 to 2001, he was with Alcatel Shanghai Bell Corporation, as a Software Engineer in the Mobile Communication Department. He had been involved in mobile network systems research and development. He was a Research Assistant with the Communications and Signal Processing Applications Laboratory at Ryerson University from 2002 to 2004. Since January 2005, he has been with the Multimedia Compression Laboratory at Queens University, Kingston, ON. His research interests include image processing and robust audio coding.

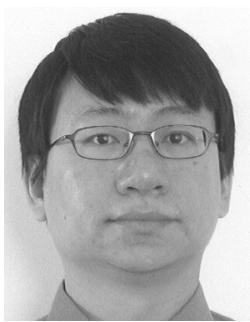

Xiao-Ping Zhang (M'97-SM'02) received the B.S. and $\mathrm{Ph} . \mathrm{D}$. degrees in electronic engineering from Tsinghua University, China, in 1992 and 1996, respectively.

Since Fall 2000, he has been with the Department of Electrical and Computer Engineering, Ryerson University, Toronto, ON, Canada, where he is now an Associate Professor and Director of the Communication and Signal Processing Applications Laboratory (CASPAL). Prior to joining Ryerson, from 1996 to 1998, he was a postdoctoral fellow at the University of Texas, San Antonio, and then at the Beckman Institute, University of Illinois at Urbana-Champaign, Urbana. He held research and teaching positions at the Communication Research Laboratory, McMaster University, Hamilton, ON, in 1999. From 1999 to 2000, he was a Senior DSP Engineer at SAM Technology, Inc., San Francisco, and a Consultant at the San Francisco Brain Research Institute. His research interests include signal processing for communications, multimedia data hiding, retrieval and analysis, computational intelligence, and various applications in bioengineering and bioinformatics.

Dr. Zhang received the Science and Technology Progress Award from the State Education Commission of China for his significant contribution in a National High-Tech Project in 1994. He is a registered Professional Engineer in Ontario, Canada, and the publicity Co-Chair for the ICME'06 and program Co-Chair for ICIC'05. 